\title{
BMJ Open Use of implantable meshes for augmented rotator cuff repair: a systematic review and meta-analysis
}

\author{
Mathew Baldwin (D) , ${ }^{1}$ N S Nagra, ${ }^{1}$ Gemma Greenall, ${ }^{1}$ Andrew J Carr, ${ }^{1}$ \\ David Beard, ${ }^{1} \mathrm{~J}$ L Rees, ${ }^{1}$ Amar Rangan, ${ }^{1,2}$ Naomi Merritt, ${ }^{1}$ Melina Dritsaki, ${ }^{3}$ \\ Sally Hopewell, ${ }^{3}$ Jonathan Alistair Cook (D) ${ }^{1}$
}

To cite: Baldwin M, Nagra NS, Greenall G, et al. Use of implantable meshes for augmented rotator cuff repair: a systematic review and meta-analysis. BMJ Open 2020;10:e039552. doi:10.1136/ bmjopen-2020-039552

- Prepublication history and additional material for this paper are available online. To view these files, please visit the journal online (http://dx.doi. org/10.1136/bmjopen-2020039552).

Received 18 April 2020 Revised 03 September 2020 Accepted 14 October 2020

A) Check for updates

(C) Author(s) (or their employer(s)) 2020. Re-use permitted under CC BY-NC. No commercial re-use. See rights and permissions. Published by BMJ.

${ }^{1}$ Nuffield Department of Orthopaedics, Rheumatology and Musculoskeletal Sciences, Oxford University, Oxford, UK ${ }^{2}$ Department of Orthopaedics, James Cook University Hospital, Middlesbrough, UK

${ }^{3}$ Centre for Statistics in

Medicine, Oxford University, Oxford, UK

Correspondence to Dr Mathew Baldwin; matthew.baldwin@ndorms.ox. ac.uk

\section{ABSTRACT}

Objective To appraise studies reporting on clinical effectiveness and safety of surgical meshes used to augment rotator cuff repairs (RCRs).

Design Systematic review and meta-analysis.

Data sources MEDLINE, Embase and Cochrane databases were searched between April 2006 and April 2020.

Eligibility criteria All studies evaluating adults $(\geq 18$ years) undergoing RCR were considered. There were no language restrictions.

Data extraction and synthesis Screening, data extraction and quality appraisal were conducted by two independent reviewers. Meta-analysis was conducted using a random-effects models if $\geq 2$ comparative studies reported the same outcome measure. Risk of bias assessment was undertaken for randomised (RoB2, Cochrane) and comparative studies (ROBINS-I, Cochrane).

Results We included 60 studies, consisting of 7 randomised controlled trials, 13 observational comparative studies and 40 observational case series. All comparative studies reported on shoulder-specific functional outcome scores, 18 on the radiographic occurrence of re-tear and 14 on pain score metrics. All studies contained some risk of bias.

Compared with non-augmented repair, a small improvement in shoulder-specific function or pain scores was observed for synthetic patches with a mean improvement of 6.7 points on the University of California Los Angles (UCLA) shoulder score (95\% Cl 0.1 to 13.4) and 0.46 point reduction on the Visual Analogue Scale $(95 \%$ $\mathrm{Cl}-0.74$ to -0.17$)$, respectively. A reduced likelihood of radiologically observed re-tear was observed for synthetic (risk ratio (RR) $0.41,95 \% \mathrm{Cl} 0.27$ to 0.61 ) and allograft (RR $0.34,95 \% \mathrm{Cl} 0.18$ to 0.65$)$ patches. A total of 49 studies reported on the occurrence of complications. Slightly higher crude complication rates were observed following patch-augmented repair (2.1\%) than standard repair (1.6\%).

Conclusions While several studies suggest a decreased failure rate and small improvements in shoulder function and pain following augmented RCR, a paucity of rigorous clinical evaluation, for both effectiveness and safety, prevents firm recommendations.

Prospero registration number CRD42017057908.
Strengths and limitations of this study

- The largest systematic appraisal of the clinical effectiveness and safety of implantable meshes for augmented rotator cuff repair.

- Thorough searching of three major electronic databases and reporting as per Preferred Reporting Items for Systematic Reviews and Meta-Analysis guidelines. The study protocol was published a priori, with inclusion of all non-English language articles.

- Study bias and substantial heterogeneity between studies means that our meta-analysis results must be interpreted very cautiously, seriously limiting our ability to draw firm recommendations.

- The observed differences in outcomes between patch types could reflect, to some degree, chance findings given the limited numbers of studies and small typical study size. Confirmation of findings by further trials is warranted.

\section{INTRODUCTION}

Shoulder pain is the third most prevalent musculoskeletal disorder and is responsible for prolonged periods of disability, absence from work and a significant healtheconomic burden. ${ }^{1-3}$ Rotator cuff problems account for a large proportion of shoulder pain and results in pain, weakness, reduced shoulder mobility and sleep disturbance. ${ }^{4}$ It is estimated that the overall prevalence of full-thickness tears is between $15 \%$ and $20 \%$ with the rate set to increase as populations age ${ }^{56}$ While some are asymptomatic, many symptomatic full-thickness tears will often require surgical repair, with successful repair correlating with symptom resolution. ${ }^{7}$ Indeed, 9000 rotator cuff repairs (RCRs) are performed each year in the NHS in England alone, at a cost of $£ 6500$ per operation. ${ }^{8}$ Unfortunately, randomised controlled trials (RCTs) have demonstrated a failure rate of up to $40 \%$, with increasing patient age and tear size, both predictive of failure. ${ }^{79}$ While various surgical techniques have attempted to 
improve the outcome of RCR, there remains a real need to improve healing rates.

One approach is the use of an implant called a surgical mesh, or patch, to augment the repair. A patch made from Teflon was first described over 30 years ago, ${ }^{10}$ but recently, the number and types of patch specifically for rotator cuff surgery have increased significantly. These can broadly be divided into categories based on the materials used; xenograft or allograft are decellularised extracellular matrix derived from animal or human cadaveric tissues, respectively; synthetic grafts are materials derived from a variety of bio-inert polymers; and autografts are patient's own whole tendon harvested from various anatomical sites. Patch augmentation can also be classified based on the method of application into 'on-lay' or 'bridging'. The former refers to the application of a synthetic or biological patch over the top of a standard repair to provide mechanical stability and biological stimulation, reducing the likelihood of failure and improving patient outcomes. ${ }^{1112}$ In contrast, 'bridging' refers to the use of a patch as an interposition graft to fill any residual defect of an otherwise irreparable tear, providing a scaffold for the regeneration of tendon and/or scar tissue formation. $^{13}$

Recent and important debate surrounding medical device regulation has emphasised the need for robust evaluations of safety, efficacy and survellience. ${ }^{14}$ Unfortunately, in the context of patch augmentation, the growing number of available patches, mixed results and recent concerns over safety, including adverse immunological responses, ${ }^{15}$ have generated a clouded and uncertain landscape.

The aim of this systematic review is to identify and critically appraise those studies reporting on the clinical effectiveness and safety of patch-augmented surgical repair in adults with rotator cuff tears.

\section{METHODS}

\section{Protocol and registration}

The review protocol and search strategy has been previously been registered (PROSPERO Registration: CRD42017057908) and published in full. ${ }^{16}$

\section{Eligibility criteria}

\section{Population}

The review incorporated studies of adult ( $\geq 18$ years) patients who required surgical repair of a rotator cuff tear. No restrictions were applied to tear type (partial or full thickness), size (small through to massive), tendon involvement (supraspinatus, infraspinatus, teres minor or subscapularis), primary or recurrent tears, or the presence of medical comorbidities. For the purpose of this review small $(<1 \mathrm{~cm})$, medium $(1-3 \mathrm{~cm})$ and large $(3-5$ $\mathrm{cm})$ tears were classified according to the DeOrio and Cofield classification. ${ }^{17}$ Due to the large number of classification systems available, tears were considered massive if they met one of following criteria: (1) Measured $>5 \mathrm{~cm}$ in the anterior-posterior dimension, ${ }^{17}$ (2) Involved $\geq 2$ tendons,${ }^{18}$ or were (3) Described as being massive by the study authors.

\section{Interventions}

All studies where at least one treatment arm included the use of patches to augment rotator cuff surgery were included. A patch was defined as an implantable human, synthetic or animal material which is used with the aim of improving tissue healing and/or patient outcome via some form of mechanical support. Patches were grouped into xenograft, allograft, autograft or synthetic. There was no restriction placed on the type of surgery received or the experience of the surgeon. The type of patch surgery was classified as either (1) 'on-lay' or (2) 'bridging' in accordance with previously reported definitions. ${ }^{19}$ We excluded studies that investigated the use of sutures or anchors in isolation, or studies investigating drug therapy or physiotherapy, except when used as a comparator group or in addition to patch augmentation.

\section{Comparators}

No restriction was placed on the type or number of control groups.

\section{Outcomes}

The primary outcomes of interest in this review were: (1) Shoulder-specific function and pain scores-measured using a previously validated scale. (2) Patch-related adverse events (complications). (3) Shoulder pain outcomes-measured using validated tools such as the Visual Analogue Scale (VAS) or other scales. (4) Healthrelated quality life-measured using tools such as Short Form-36 (SF-36), EuroQol 5-dimension (EQ-5D) Questionnaires or other assessment measures. The main secondary outcome was the radiological assessment of postoperative rotator cuff integrity (re-tear).

\section{Study types}

We considered all relevant RCTs and observational studies (comparative and single group) involving at least five patients. No language restrictions were applied. In vitro studies, animal studies, review articles, editorials and studies involving up to five patients were all excluded.

\section{Search strategy}

A previous Cochrane review had carried out a comprehensive search prior to April 2006. ${ }^{20}$ We searched the following databases between the dates of April 2006 and February 2017 (and updated our search in April 2020): (1) MEDLINE, (2) Embase, (3) The Cochrane Library. In addition, the reference list of all identified articles and reviews identified were checked for relevant articles. ${ }^{1921-24}$

\section{Study selection}

Two authors (MB and NSN) independently screened all titles and abstracts identified from the search strategy. Full reports for all relevant studies identified were then reviewed and assessed against the eligibility criteria. A 


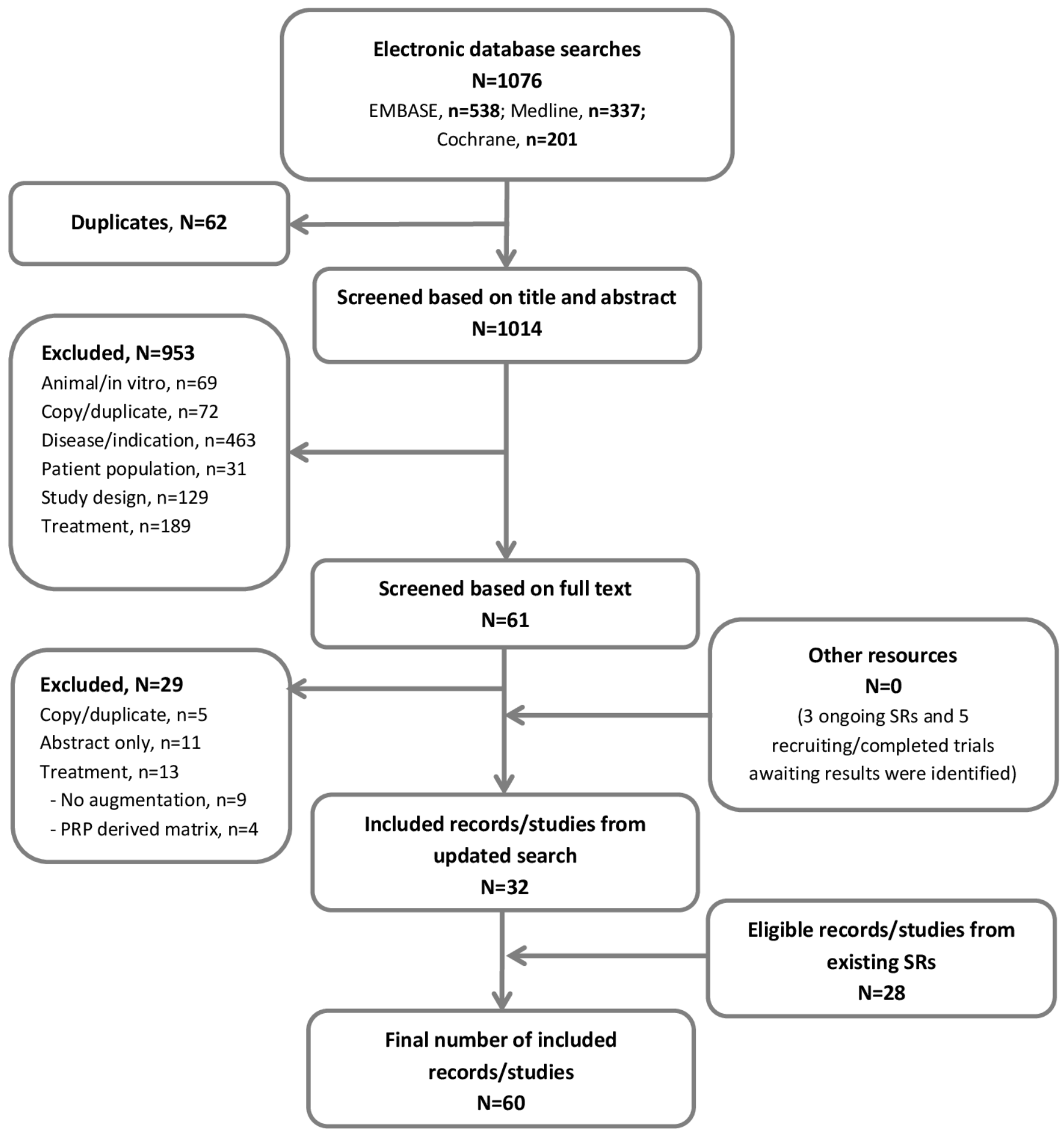

Figure 1 PRISMA flow chart of study selection. N, number; PRISMA, Preferred Reporting Items for Systematic Reviews and Meta-Analyses; SR, systematic review.

third independent reviewer (GG) was available to resolve any disagreements regarding study inclusion. Reasons for exclusion are detailed in the Preferred Reporting Items for Systematic Reviews and Meta-Analysis (PRISMA) flow diagram (figure 1).

\section{Data extraction}

Two authors (MB and NSN) extracted the following data from all eligible studies: general study information (authors, publication year, study location), study population (sample size, age, gender, tear size), study characteristics (study design, inclusion/exclusion criteria, duration of clinical and radiological follow-up, surgical technique, patch characteristics), all primary and secondary outcomes for each study, and adverse events or complications. Each reviewer independently checked the results of the data extraction process. 


\section{Risk of bias assessment}

The risk of bias was independently assessed by two authors (MB and NSN) and discrepancies discussed with a third reviewer (GG) allowing resolution based on unanimous decision. RCTs were assessed using the isk-of-bias tool for randomized trials (RoB2) provided by the Cochrane Collaboration. ${ }^{25}$ Observational comparative studies were assessed using the ROBINS-I tool (Risk Of Bias In Nonrandomised Studies - of Interventions). ${ }^{26}$

\section{Data analysis}

Identified studies were stratified (RCTs, observational comparative studies, single-arm studies) and a narrative summary of results from RCTs and observational comparative studies reported in accordance with the standards set out in the PRISMA-P checklist. ${ }^{27}$ Data from single-arm studies were only used in the quantification of complications. All studies that compared the outcomes of RCR with graft augmentation versus standard RCR were considered for meta-analysis. A meta-analysis was conducted only for outcomes consistently reported across studies and forest plots constructed using the R (V.3.2.4) packages 'meta' and 'metafor'. Regardless of the observed statistical heterogeneity, we conducted an analysis for each patch type (xenograft, allograft, autograft or synthetic) when each type was represented by at least two studies. Given the known controversy surrounding xenograft isolated from small intestinal submucosa (SIS), the analysis for xenografts was further divided into SIS-derived and non-SIS. There were insufficient study numbers to permit subgrouping based on graft configuration (on-lay or bridging).

\section{Statistical analysis}

For dichotomous parameters included in the metaanalysis the risk ratio (RR) with $95 \%$ CI was calculated for each graft type. For continuous variables, such as shoulder-specific functional outcome scores, the effect was reported as the mean difference with $95 \%$ CI. Due to significant heterogeneity in the specific functional shoulder scores used between studies, a meta-analysis was conducted using the most frequently used score across all studies at final follow-up. Within each patch type, if no single functional outcome score was consistently used, we combined scores and the standardised mean difference was meta-analysed (95\% CI). Studies in which no SD was calculable, or where only subcomponents of functional outcome scores were reported, were excluded. Heterogeneity was characterised by use of the $I^{2}$ statistic and a random-effect analysis was used to allow for heterogeneity among studies. Meta-analyses were conducted on available data across all comparative studies; sensitivity meta-analyses were then performed to assess the impact of restricting to only one study design where two or more studies of the same design were available.

\section{Patient involvement}

Patient representatives were full members of the PARCS Study $^{28}$ steering committee and provided critical feedback on the systematic review protocol.

\section{RESULTS}

\section{Study selection}

The search strategy identified 1076 articles, of which 62 were duplicates (figure 1). A total of 1014 abstracts were reviewed in detail with 61 appearing to meet inclusion criteria. After full-text review 29 articles were excluded (details in figure 1). A further 28 articles were identified from existing systematic reviews, generating a total of 60 studies for inclusion, including 2 non-English language articles. The summaries below focus predominantly on the comparative (RCTs and observational) studies.

\section{Study characteristics}

Seven RCTs and 13 observational comparative studies analysing 1128 patient events were identified. Most comparative studies assessed a single patch against standard repair, with some studies having up to three treatment arms. ${ }^{29-31}$ A single study compared autograft patch augmentation to conservative therapy, ${ }^{32}$ while a further study assessed the effect of xenograft patch with or without mesenchymal stem cell augmentation. ${ }^{33}$ The trial of mesenchymal stem cell augmentation by Lamas et al was terminated early due to safety concerns ${ }^{33}$ as was the study by Walton et al which assessed the RESTORE patch. ${ }^{34}$ Study population sizes ranged from 13 to 105 patients for RCTs (age range 29-85 years), and 9 to 152 patients (age range 36-83 years) among observational comparative studies, with a predominance of male participants across all studies. Only two studies included the full spectrum of full thickness tear sizes, with most studies instead restricting recruitment to large or massive tears of the supraspinatus and infraspinatus (table 1). Other eligibility criteria were highly heterogeneous (online supplemental table 1).

\section{Surgical characteristics}

Across all the comparative studies a total of 15 different patches was used. Decellularised xenograft patches were the most commonly investigated $(n=9$; Restore $\mathrm{n}=4$ ). Surgical techniques could be classified as fully arthroscopic $(55 \%, \mathrm{n}=11)$, open $(35 \%, \mathrm{n}=7)$ or a mixture of both $(10 \%, \mathrm{n}=2)$. Regarding the method of patch utilisation, a larger proportion of studies investigated an 'on-lay' $(60 \%, \mathrm{n}=12)$ rather than a 'bridging' $(40 \%, \mathrm{n}=8)$ technique.

\section{Risk of bias}

Assessment of bias was conducted for all RCTs and comparative studies (online supplemental tables 2 and 3 ). For the study by Bryant et $a \ell^{35}$ some concerns over bias were identified but with the remaining RCTs assessed as having a high risk. These findings are based 


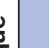

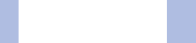

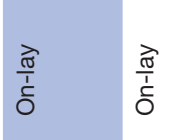

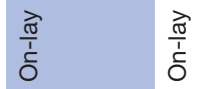

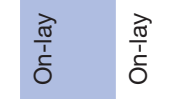
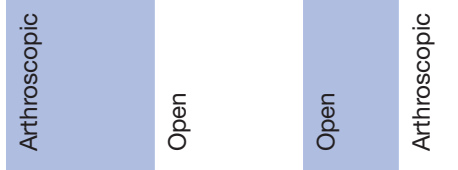

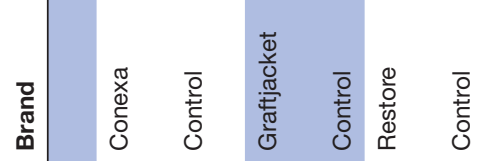

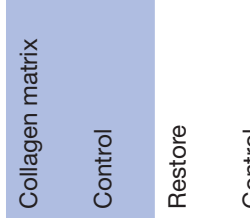

占

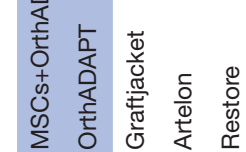

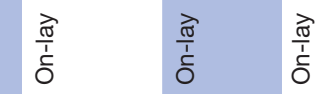
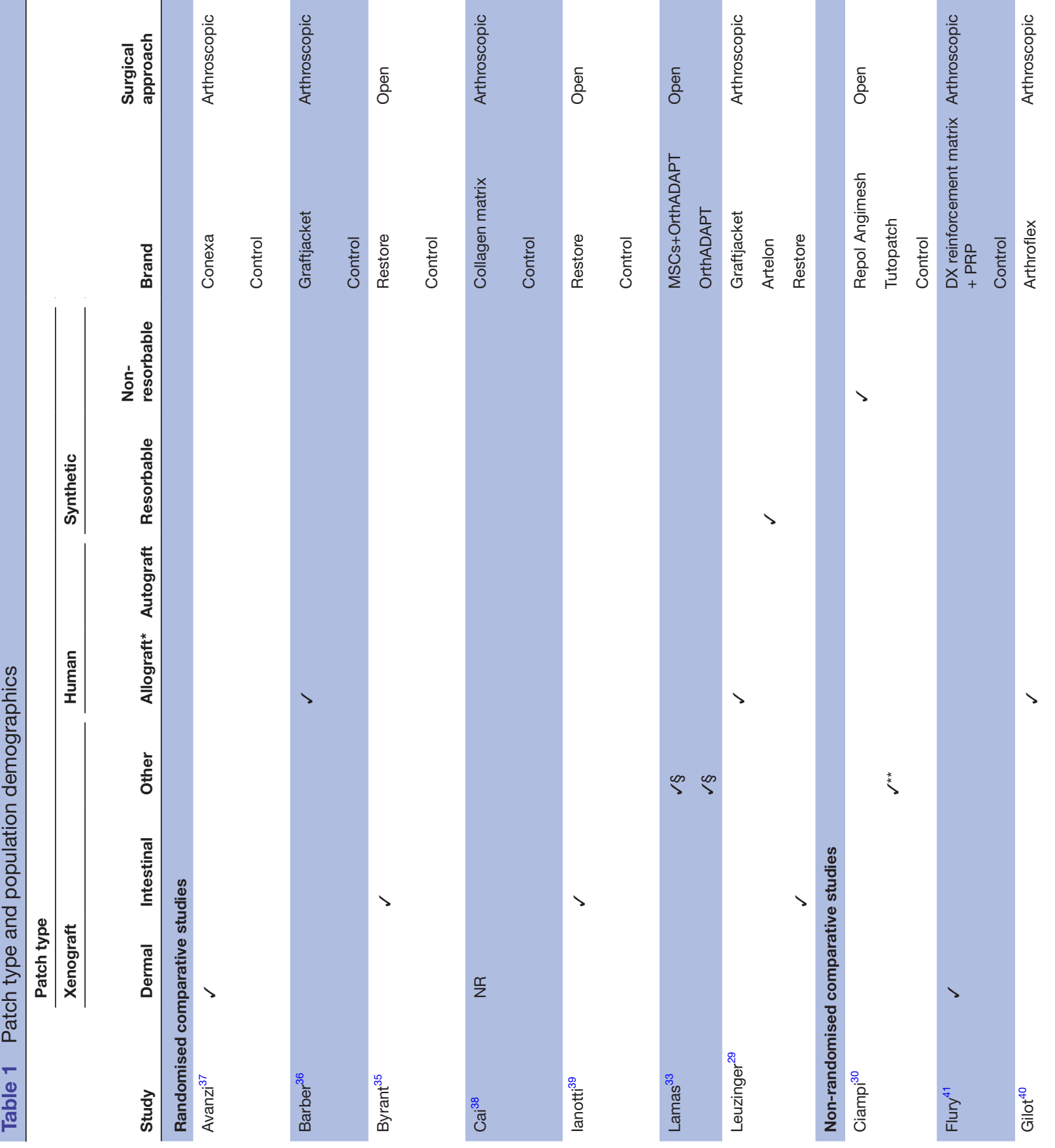

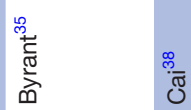

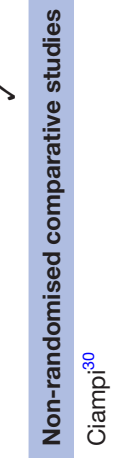

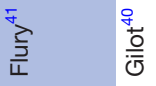




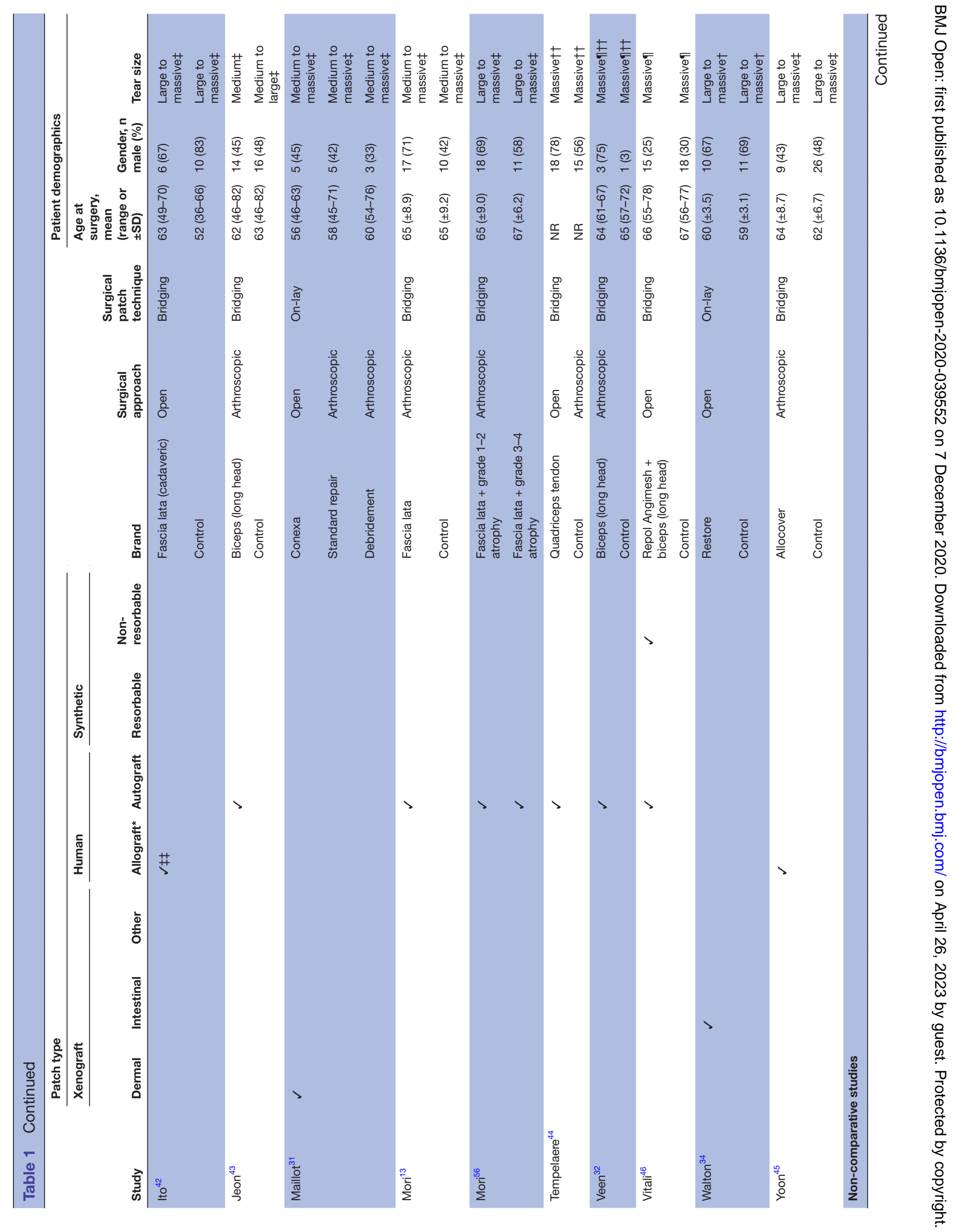




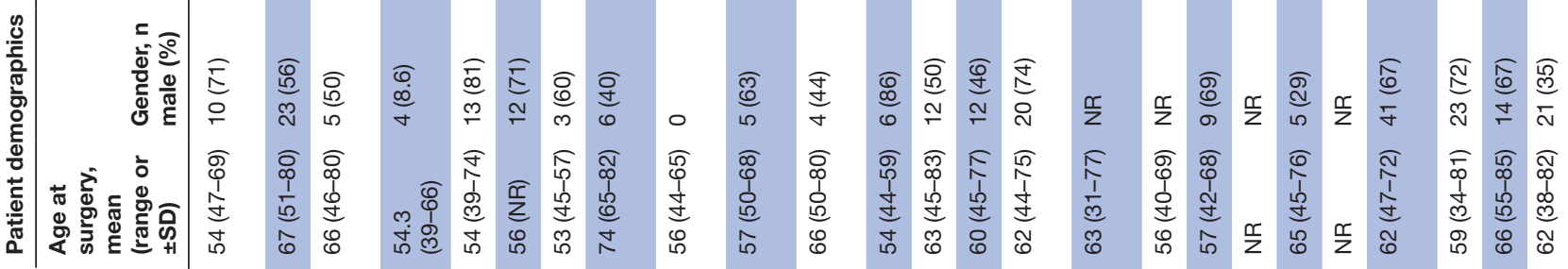

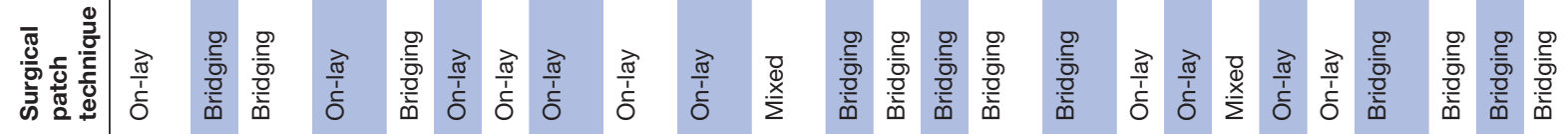

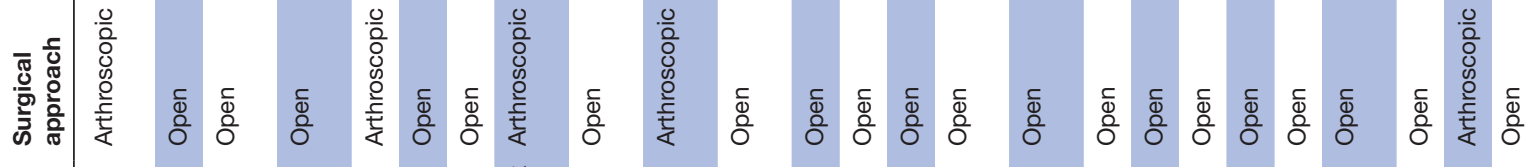

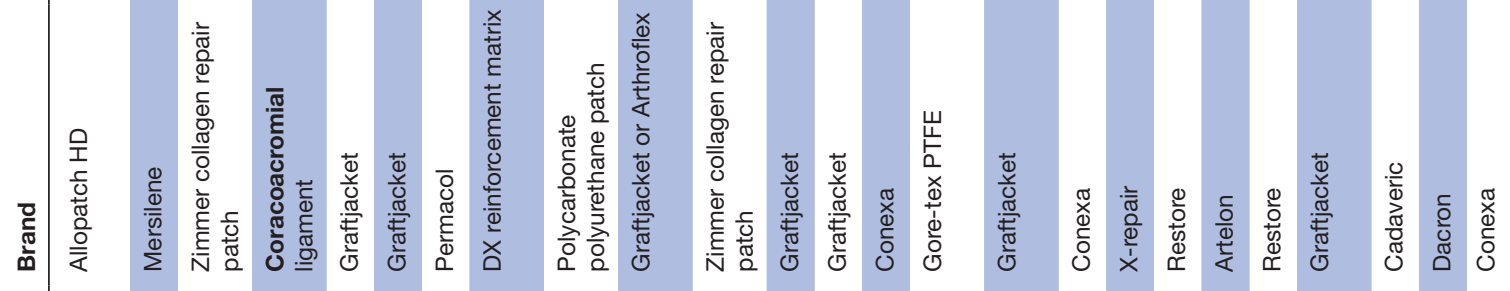

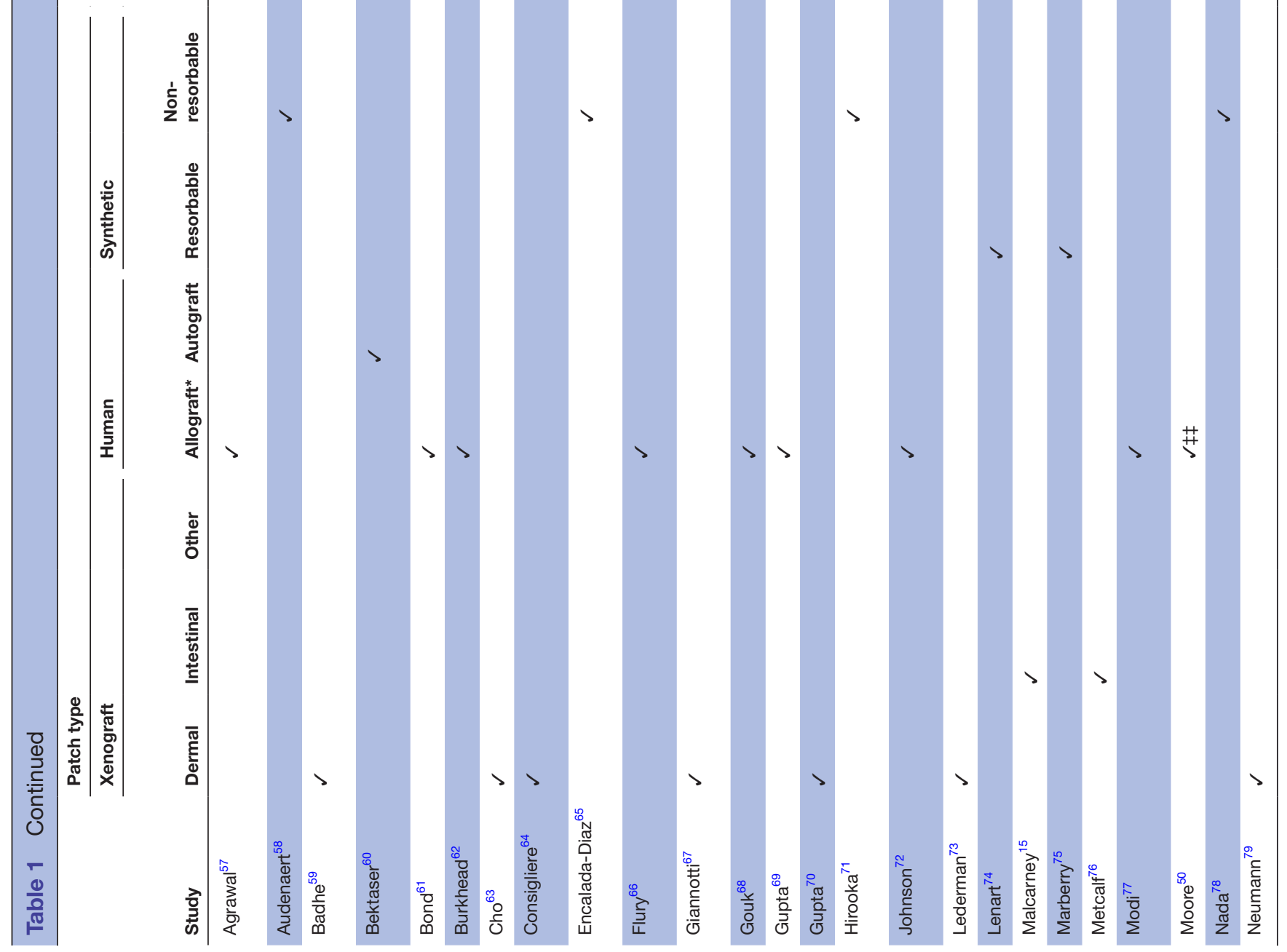




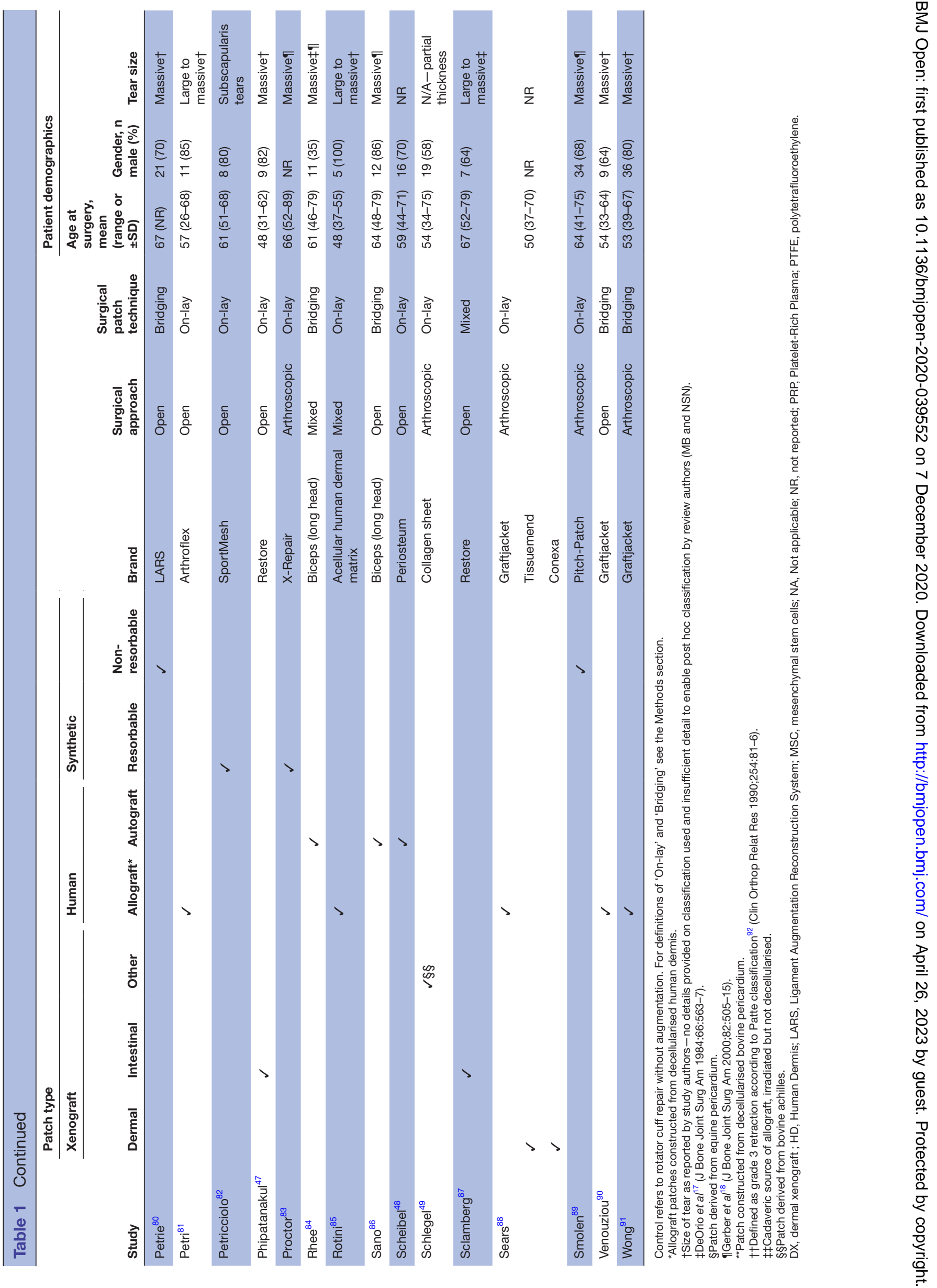


on inadequate randomisation methodologies and a lack of study methodology detail, in particular surrounding blinding of patients and outcome assessors. All observational comparative studies had a serious risk of bias, centring around the potential for confounding, bias in patient selection and outcome measurement.

\section{Outcomes summaries}

Shoulder-specific function and pain scores

Eleven different outcomes scores were used to assess shoulder-specific function and pain (online supplemental table 1). The Constant Scale (70\%), American Shoulder and Elbow Surgeons (ASES) Score (40\%) and University of California Los Angles (UCLA) Scale (30\%) were most commonly reported in the comparative studies with most studies reporting multiple functional scores.

Among RCTs, only one study found a sustained, statistically meaningful improvement in ASES and Constant Scores, but not UCLA Scale, 2 years after implantation of an allograft patch (online supplemental table 4). ${ }^{36}$ A further two studies, investigating a dermal xenograft $(\mathrm{DX})^{37}$ and an unspecified non-proprietary patch, ${ }^{38}$ reported initial improvements in Constant Scores at 12 months, but this was not sustained at longer (2 years) follow-up. The two RCTs investigating decellularised porcine small intestine submucosa (Restore) ${ }^{35} 39$ failed to demonstrate an improvement in patient-reported outcomes at 1-2 years follow-up, while the study by Leuzinger $e t a l^{29}$ only undertook intragroup comparisons between preoperative and postoperative Constant Scores, reporting similar improvements following implantation of an allograft, xenograft or synthetic patch.

For the non-randomised comparative studies, only three reported a significant improvement in functional shoulder scores for synthetic, ${ }^{30}$ human allograft ${ }^{40}$ and fascia lata autografts. ${ }^{13}$ The remaining studies found no significant improvement, ${ }^{31} 3441-45$ while the studies by Ito $e t a l l^{42}$ Veen $e t a l^{2}$ and Vitali $e t a l^{46}$ did not undertake intergroup comparisons.

\section{Repair failure}

Integrity of the surgical repair was assessed by all RCTs and $11(84 \%)$ observational comparative studies, with a re-tear rate ranging from $2 \%$ to $100 \%$ following patch implantation and $13 \%$ to $65 \%$ following a standard RCR (online supplemental table 5). MRI was the the most common imaging modality (72\%) used to diagnose re-tears, with an MR arthrogram used in a further $17 \%$ of studies. The majority of studies $(72 \%)$ undertook postoperative imaging after 1-2 years but with considerable heterogeneity existing in the radiological classification of re-tears and with seven studies ${ }^{30} 323338404246$ not providing any details. While the RCTs investigating human allograft (Graftjacket), ${ }^{36}$ DX (Conexa) ${ }^{37}$ and an unspecified collagen patch, ${ }^{38}$ each demonstrated a significantly lower failure rate in the augmentation arm, neither of the RCTs investigating the small-intestine submucosa xenograft patch (Restore) ${ }^{35} 39$ found any reduction in re-tear rate.
In conflict with these findings, a multipatch comparative study ${ }^{29}$ found no difference in failure rate between xenograft (Restore) and two different patches; human allograft (Graftjacket) or synthetic (Artelon). Among the observational comparative studies, significantly lower rates of re-tears were reported with augmentation using synthetic (Repol Angimesh), ${ }^{30}$ autograft (fascia lata) ${ }^{13}$ or allograft patches (Arthroflex and Allocover) ${ }^{40} 45$ while no improvement in re-tears was observed following augmentation with DX (reinforcement matrix), ${ }^{41}$ long head of biceps tendon autograft ${ }^{43}$ or for the Restore ${ }^{34}$ patch.

\section{Pain scores}

Only two studies (Gilot et al, Athroflex; ${ }^{41}$ Mori et al, fascia lata ${ }^{13}$ ) reported significant reduction in pain when compared against standard repair (online supplemental table 6). Interestingly, the study by Walton et al, who used a 'mean activity pain score', found an increase in pain for the first 3 months following implantation of the Restore patch, which subsequently normalised by 6 months. ${ }^{34}$ The remaining 10 studies either did not report intergroup comparisons $(\mathrm{n}=5),{ }^{30} 32334246$ or found no significant difference in pain scores between treatment arms $(\mathrm{n}=5) . .^{39} 43-45$

\section{Quality of life}

Only five comparative trials reported the use of either the SF-12, SF-36 or EQ-5D Scores (online supplemental table 7). When compared with standard repair, two RCTs investigating porcine SIS xenograft (Restore) found no difference in the physical or mental components of SF-36. ${ }^{35} 39$ Similarly, an RCT (Avanzi et $a l)^{37}$ and an observational comparative study (Flury et $a l)^{41}$ assessing different DXs did not find an improved EQ-5D at 2-year follow-up. Conversely, a comparative study using human allograft (Athroflex) reported a significant improvement in all components of SF-12 at 6 months and 2 years postoperatively (though not at 3 months). ${ }^{40}$

\section{Complications}

Forty-nine studies provided data on complications, of which 24 studies reported the occurrence of 83 complications in a total population of 1567 patients undergoing any form of augmentative surgery and 488 patients receiving a standard RCR (online supplemental table 5). The overall crude complications rates were $4.5 \%$ for patients undergoing any form of patch augmentation and $1.6 \%$ following non-augmentative surgery. However, by excluding six studies in the augmentation group which had particularly high rates of complications (20\%-74\%) following quadriceps tendon, MSC seeded xenograft, Restore patch or humeral periosteal augmented repair, ${ }^{33} 3439444748$ the overall rate of complications following patch augmentation was $2.6 \%$. An inflammatory response was recorded in 18 patients (all reported complications are detailed in online supplemental table 5). The majority of these events $(n=11)$ occurred in patients who received an SIS xenograft (Restore) patch, but with reactions also 
A Forest plot comparing shoulder specific pain and function outcome scores at final follow-up between autograph patches and standard repair

\begin{tabular}{|c|c|c|c|c|c|}
\hline \multirow{3}{*}{ A } & \multirow{2}{*}{\multicolumn{2}{|c|}{$\begin{array}{l}\text { Forest plot comp } \\
\text { Human Autograft }\end{array}$}} & \multirow{2}{*}{\multicolumn{3}{|c|}{$\begin{array}{l}\text { ulder specific p } \\
\text { Standard Repair }\end{array}$}} \\
\hline & & & & & \\
\hline & Mean & SD & Total & Mean & SD \\
\hline Jeon 2017 & 88.20 & 6.90 & 31 & 87.40 & 7.20 \\
\hline Mori 2013 & 94.10 & 5.40 & 24 & 85.70 & 14.10 \\
\hline
\end{tabular}
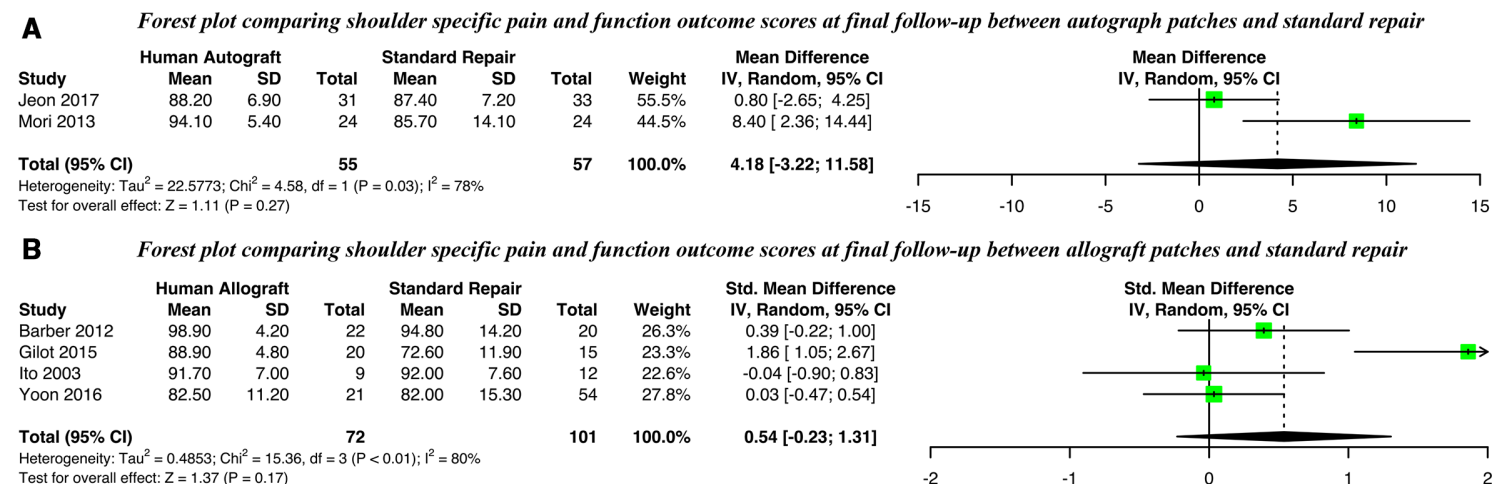
Test for overall effect: $Z=1.37(P=0.17)$

\begin{tabular}{|c|c|c|c|c|c|}
\hline \multicolumn{3}{|c|}{ Xenograft(non-SIS) Patch } & \multicolumn{3}{|c|}{ Standard Repair } \\
\hline Study & Mean & SD & Total & Mean & SD \\
\hline Ciampi 2014 & 14.70 & 2.00 & 49 & 14.90 & 2.00 \\
\hline Malliot 2018 & 75.80 & 8.60 & 11 & 74.70 & 4.30 \\
\hline Avanzi 2019 & 95.50 & 5.50 & 38 & 92.60 & 9.30 \\
\hline Flury 2018 & 24.90 & 11.00 & 19 & 27.50 & 16.50 \\
\hline
\end{tabular}

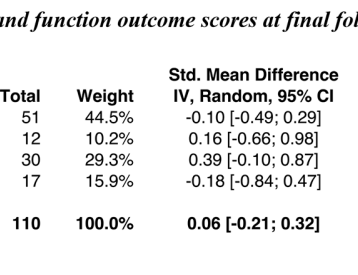

low-up between xenografts (non-small intestine submucosa)

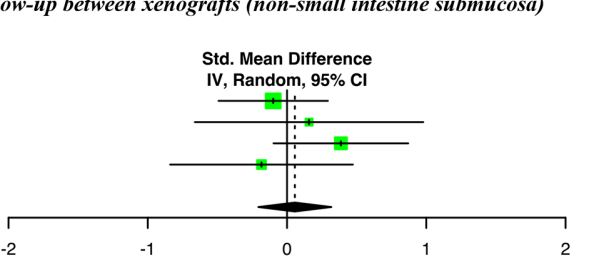

D Forest plot comparing shoulder specific pain and function outcome scores at final follow-up between synthetic patches and standard repair

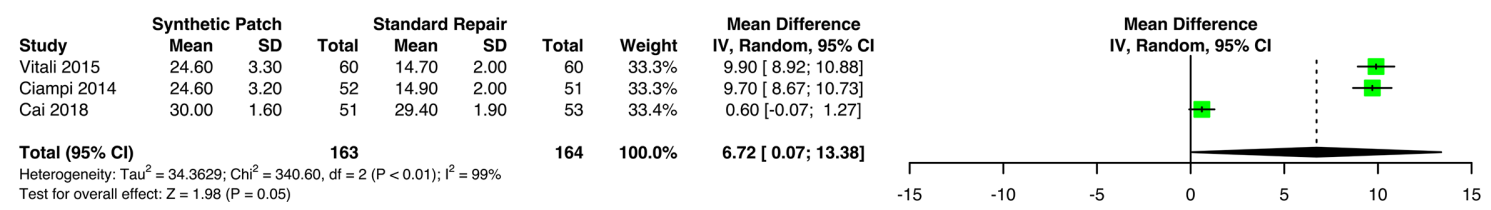

Figure 2 Forest plots comparing shoulder-specific functional outcomes scores at final follow-up for (A) Autografts, (B) Allografts, (C) Xenografts (non-SIS) or (D) Synthetic patches against standard repair alone. SIS, small intestine submucosa; IV, Random, a random-effects meta-analysis is applied, with weights based on inverse variances.

reported after implantation of bovine-derived, ${ }^{49}$ equinederived ${ }^{33}$ irradiated ${ }^{50}$ or decellularised ${ }^{29}$ human allograft and synthetic patches. ${ }^{29}$ Excluding all adverse events concerning the Restore patch, which has been withdrawn from the marketplace, the crude complication rate for patches in current clinical use was $2.1 \%$.

\section{Meta-analysis}

Shoulder-specific function and pain

Of the 20 comparative studies, 12 provided sufficient data on postoperative functional outcome scores to be included in the meta-analysis (figure 2). An improvement on the UCLA shoulder scale was observed for synthetic patches at long-term (range 24-36 months) follow-up (mean difference $6.72,95 \%$ CI 0.07 to 13.38 ) but not in the ASES Score of studies of autografts (mean difference $4.18,95 \%$ CI -3.22 to 11.58 ). Studies of allografts or xenografts derived from dermis or pericardium (non-SIS) used differing measures but with no significant standardised mean differences observed for either. Level of heterogeneity, across all patch types, was generally very high $\left(\mathrm{I}^{2}\right.$ $>70 \%$ ). Insufficient data were available for xenografts derived from intestinal submucosa (SIS). Sensitivity analyses did not find any impact of study design (randomised vs observational) except for synthetic patches where the sole RCT differed from the observational studies (online supplemental figure 1).

\section{Re-tear rate}

Fourteen studies were included in a meta-analysis for re-tear rate (figure 3). A significantly lower re-tear rate was seen for allograft patches (RR $0.34,95 \%$ CI 0.18 to 0.65 ) and synthetic patches (RR $0.41,95 \%$ CI 0.27 to 0.61 ) but not for autografts, SIS-derived or non-SIS-derived xenografts (there was substantial heterogeneity for the latter, $\mathrm{I}^{2}=67 \%$ ). Meta-analysis results were not influenced by study design (online supplemental figure 2).

Pain

Eight observational comparative studies had sufficient data for a meta-analysis of postoperative pain (figure 4). A small, possibly non-clinically significant, ${ }^{51}$ improvement in postoperative pain was only observed for synthetic patches (mean difference $-0.46,95 \%$ CI -0.74 to $-0.17, \mathrm{I}^{2}=0 \%$ ). Meta-analyses of allograft, autograph and xenograph (non-SIS) patches did not show any statistically significant differences. Level of heterogeneity was generally very high $\left(\mathrm{I}^{2}>70 \%\right)$. Insufficient data were available for a meta-analysis of postoperative pain following augmentation with xenograft patches derived from SIS.

Health-related quality of life

There were insufficient data available to meta-analyse the effect of patch augmentation on quality of life. 
A

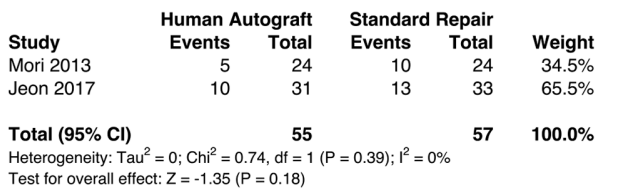

Forest plot comparing re-tear rates at final follow-up between autograft patches and standard repair

\section{Risk Ratio \\ $\mathrm{MH}$, Random, $95 \% \mathrm{Cl}$ \\ $0.50[0.20 ; 1.25]$ \\ $0.82[0.42 ; 1.59]$ \\ $0.69[0.40 ; 1.18]$}

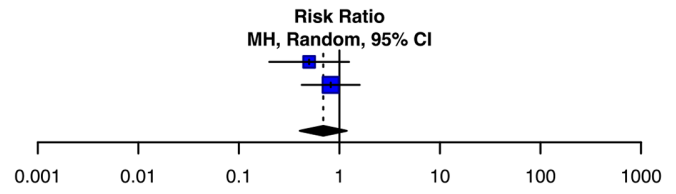

C Forest plot comparing re-tear rates at final-follow up between xenografts (small intestine submucosa) and standard repair

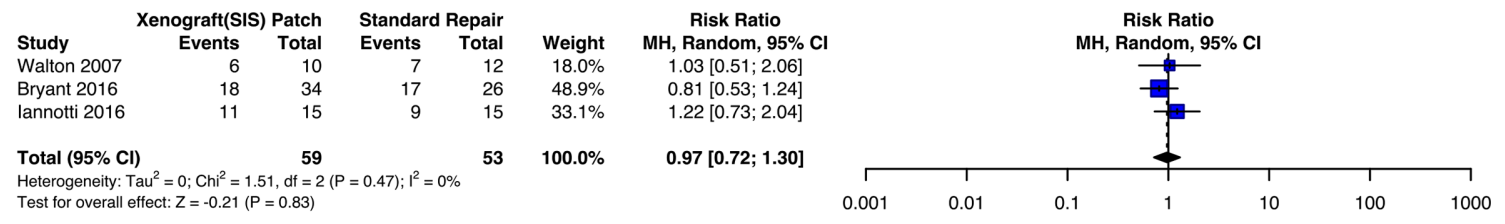

D Forest plot comparing re-tear rates at final-follow up between xenografts (non-small intestine submucosa) and standard repair

\begin{tabular}{|c|c|c|c|c|c|c|}
\hline \multicolumn{3}{|c|}{ Xenograft(non-SIS) Patch } & \multicolumn{2}{|c|}{ Standard Repair } & \multicolumn{2}{|r|}{ Risk Ratio } \\
\hline Study & Events & Total & Events & Total & Weight & $\mathrm{MH}$, Random, $95 \% \mathrm{Cl}$ \\
\hline Avanti 2019 & 1 & 13 & 8 & 18 & $17.4 \%$ & $0.17[0.02 ; 1.22]$ \\
\hline Ciampi 2014 & 25 & 49 & 21 & 51 & $48.0 \%$ & $1.24[0.81 ; 1.90]$ \\
\hline Flury 2018 & 9 & 18 & 4 & 20 & $34.6 \%$ & $2.50[0.93 ; 6.73]$ \\
\hline Total $(95 \% \mathrm{Cl})$ & & 80 & & 89 & $100.0 \%$ & $1.12[0.42 ; 3.03]$ \\
\hline $\begin{array}{l}\text { Heterogeneity: T } \\
\text { Test for overall ef }\end{array}$ & $\begin{array}{l}.4873 ; C \\
=0.23\end{array}$ & $\begin{array}{l}=6.03, \mathrm{~d} \\
0.82)\end{array}$ & $(P=0$ & $=67 \%$ & & \\
\hline
\end{tabular}

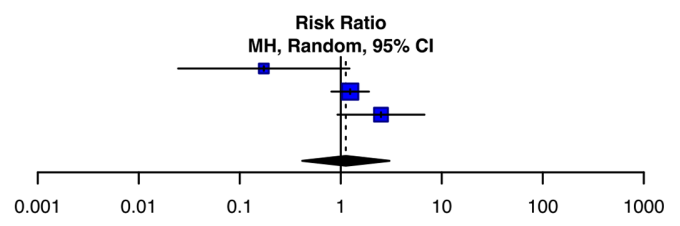

E Forest plot comparing re-tear rates at final-follow up between synthetic patches and standard repair

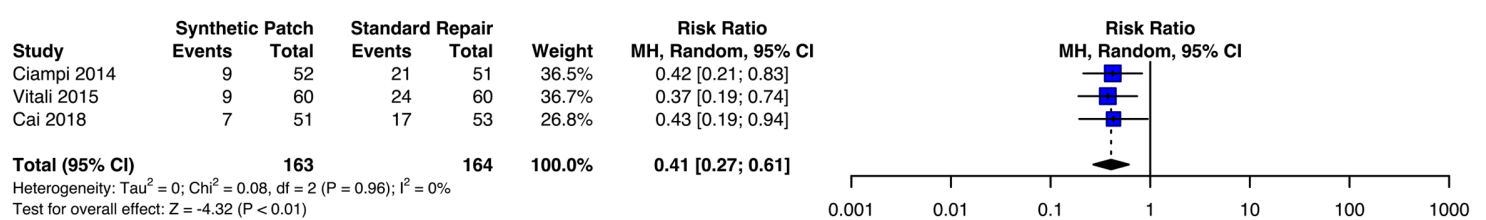

Figure 3 Forest plots comparing re-tear rates at final follow-up for (A) Autografts, (B) Allografts, (C) Xenografts (SIS) (D) Xenografts (non-SIS) or (E) Synthetic patches against standard repair alone. SIS, small intestine submucosa; MH, Random, a random-effects meta-analysis is applied, with weights based on the Mantel-Haenszel method.

\section{DISCUSSION}

The use of medical implants has recently come under increasing scrutiny. Surgical repair of the rotator cuff with patch augmentation has been proposed as a method of improving rates of tendon healing and patient outcomes. This systematic review is the largest and most comprehensive systematic appraisal of the clinical effectiveness and safety of such implants to date. Overall, the current evidence is not sufficiently robust to determine the effectiveness of patch-augmented RCR compared with standard repair alone. While this meta-analysis suggests a small improvement in pain and patient reported outcome measures (PROMS) for synthetic patches, and a moderate reduction in re-tear rate for synthetic and human allograft 
A Forest plot comparing shoulder pain at final follow-up between autograft patches and standard repair

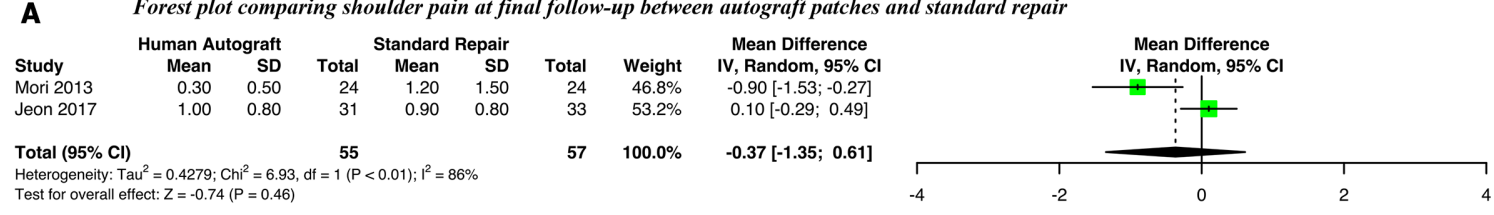

B Forest plot comparing shoulder pain at final follow-up between allograft patches and standard repair

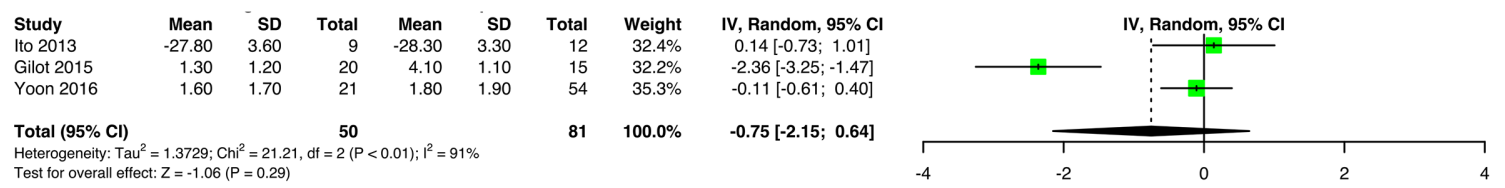

Test for overall effect: $Z=-1.06(P=0.29)$

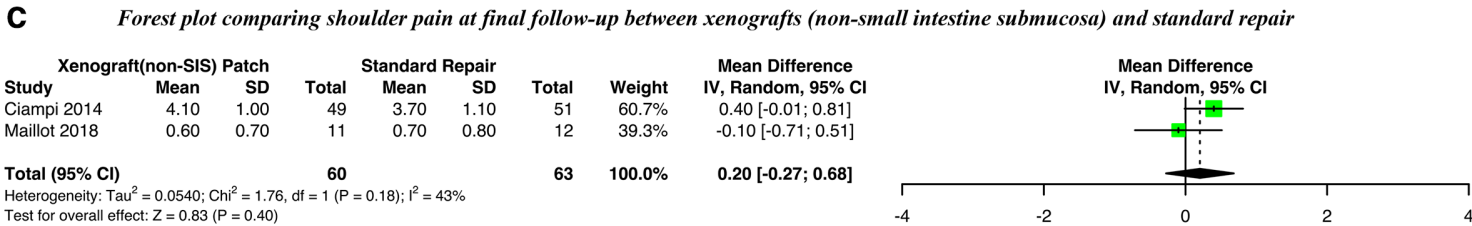

D Forest plot comparing shoulder pain at final follow-up between synthetic patches and standard repair

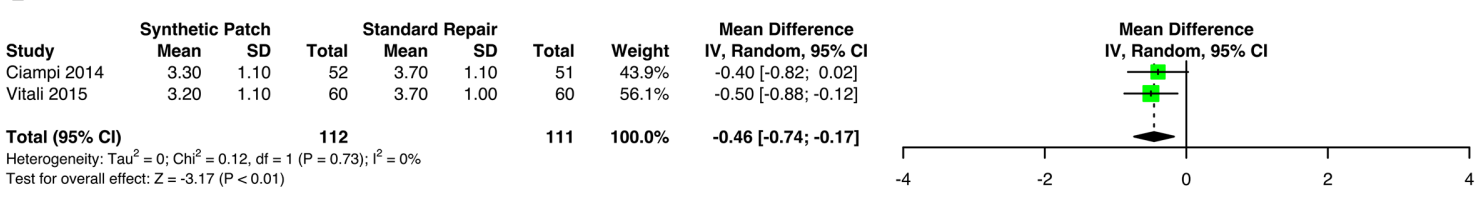

Figure 4 Forest plots comparing postoperative pain at final follow-up for (A) Autografts, (B) Allografts, (C) Xenografts (non-SIS) or (D) Synthetic patches against standard repair alone. SIS, small intestine submucosa; IV, Random, a random-effects metaanalysis is applied, with weights based on inverse variances.

patches, study bias and heterogeneity mean that these results need to be interpreted very cautiously. Further, it is unclear if the observed 6-point improvement in UCLA Score for synthetic patches is clinically meaningful. To date, the minimal clinically importance difference for UCLA shoulder score following RCR has not been established. ${ }^{52}$ However, a threshold of 30 UCLA points after 2 years has been proposed as an absolute cut-off signifying treatment success following RCR. ${ }^{53}$ In the studies investigating synthetic polypropylene patches, augmentation failed to meet this threshold. ${ }^{30}{ }^{46}$ Similarly, the 0.46-point reduction in VAS pain scores is unlikely to be clinically meaningful. ${ }^{51}$

Across 49 studies reporting complications (adverse events) with a combined population of 2055 participants, the crude complication rate was marginally higher for augmented $(2.1 \%)$ than standard $(1.6 \%)$ repairs, with specific safety concerns associated with certain patches (Restore) ${ }^{39}$ or techniques (Quadriceps allograft, Humeral Periosteal allograft, MSCs embedded in decellularised bovine pericardium). ${ }^{3344} 48$

\section{Strengths and limitations of the study}

Strengths of this this review include an a priori published protocol; ${ }^{16}$ a comprehensive search strategy; inclusion of non-English language articles and duplicate assessment of eligibility, risk of bias and data extraction. Nonetheless, there remain several limitations to the current review, which are mainly a reflection of the quality of published primary research available. Only seven RCTs have been published, of which three refer to a product (Restore) that has now been withdrawn from the market due to safety concerns, ${ }^{29} 3639$ and the study by Lamas et al was terminated due to excessive adverse events. ${ }^{33}$ Second, substantial heterogeneity between studies was observed with the majority of studies also judged to have a high risk of bias, seriously limiting our ability to draw firm recommendations. An exhaustive exploration of the heterogeneity has not been undertaken and indeed such an analysis was not declared a priori in our protocol paper. ${ }^{16}$ However, separating studies by patch type did influence the degree of heterogeneity and we would therefore recommend patch type should be considered in the design of future reviews. Finally, it should be noted that given the limited number of studies for the different patch types and the relatively small typical size of studies, the observed effects identified could reflect, to a degree, chance findings. With this in mind, the observed differences in outcomes between patch types should be interpreted cautiously and warrant confirmation by further trials.

In comparison with previous systematic reviews, ${ }^{19} 2223$ we have included four additional RCTs ${ }^{29} 333738$ and five observational comparative studies, ${ }^{32} 41-4345$ representing 324 patients, not otherwise identified. Results from our meta-analysis are, in part, consistent with a previous analysis which found an overall reduction in re-tear rate and improved ASES Scores following patch augmentation. ${ }^{22}$ The substantial number of additional studies included in this current review provide greater precision and, while 
a subgroup analysis was not originally specified in our protocol, they have allowed us to hypothesise that patch type may have an effect on patient outcomes. The occurrence of adverse events with only certain patch types adds some credibility to this notion. Previous reviews of augmented RCR have, on the basis of a presumed effect on patient outcome, excluded studies based on the size of rotator cuff tear or surgical technique (on-lay or bridging) ${ }^{19}$ It is possible that each technique reflects different patient cohorts, for example, the use of bridging scaffolds may represent larger, more chronic or even recurrent rotator cuff tears. However, we were unable to detect any overall difference in patient-reported outcomes, re-tear rate or pain scores between studies reporting on-lay or bridging techniques. It should be noted that differences in terminology makes comparison of these surgical techniques challenging-many studies use the terms irreparable, bridging, interposition or reconstruction interchangeably. To help facilitate the future interrogation of the relationship between surgical technique and outcomes we would suggest that only the terms on-lay augmentation or bridging reconstruction be used in accordance with previously published definitions. ${ }^{19}$ Similarly, a frequent lack of detail on how tear size was determined impedes any examination of tear size on treatment effectiveness. Trials should clearly describe, in a reproducible way, the classification system used when determining rotator cuff tear size.

There are a growing number of patches available for the augmentation of RCR. Despite the safety-related withdrawal of certain patches, ${ }^{34}$ as well as wider concerns surrounding medical device ${ }^{54}$ and mesh implantation, ${ }^{54}$ rigorous clinical evaluation of patch augmentation is lacking. We were particularly concerned by the absence of publicly available research for several patches currently in clinical use (eg, d-cell and Leeds-Kuff). While some studies have indicated promise for specific patches, firm recommendations in terms of patch type or surgical technique cannot be made at present. There remains a need for well-designed comparative (preferably multicentre RCTs) studies that are capable of robustly evaluating the effectiveness and safety of multiple patch types. Furthermore, routine reporting of registry data patch could address the current lack of robust safety data for cuffaugmented rotated cuff repair. ${ }^{55}$

Twitter Mathew Baldwin @mathewbaldwin3 and Jonathan Alistair Cook @ ProfJACook

Contributors JAC and AJC conceived the idea, and JAC lead the project. JLR, AR, $\mathrm{DB}, \mathrm{MD}$ and SH contributed to study design and securing funding. NM contributed to the management of the study. Article screening, data extraction, analysis and interpretation undertaken by MB, NSN and JAC. Literature searches undertaken by GG and updates by GG, MB and NSN. All authors contributed to manuscript writing while $\mathrm{MB}$ and $\mathrm{JAC}$ undertook reviewer requested revisions.

Funding The study was funded by the National Institute for Health Research (NIHR) Health Technology Assessment Programme (HTA 15/103/03), and it was supported by the NIHR-funded Oxford Biomedical Research Centre (BRC).

Disclaimer The views expressed are those of the authors and not necessarily those of the NHS, the NIHR or the Department of Health.
Competing interests AC has applied for a patent for a device which would be eligible for this type of review if it is approved for clinical use, AR has received educational and research grants from DePuy Ltd.

Patient consent for publication Not required.

Provenance and peer review Not commissioned; externally peer reviewed.

Data availability statement Data are available upon reasonable request. All data relevant to the study are included in the article or uploaded as supplementary information. The data set used and analysed for this review will be available from the corresponding author upon a reasonable request.

Supplemental material This content has been supplied by the author(s). It has not been vetted by BMJ Publishing Group Limited (BMJ) and may not have been peer-reviewed. Any opinions or recommendations discussed are solely those of the author(s) and are not endorsed by BMJ. BMJ disclaims all liability and responsibility arising from any reliance placed on the content. Where the content includes any translated material, BMJ does not warrant the accuracy and reliability of the translations (including but not limited to local regulations, clinical guidelines, terminology, drug names and drug dosages), and is not responsible for any error and/or omissions arising from translation and adaptation or otherwise.

Open access This is an open access article distributed in accordance with the Creative Commons Attribution Non Commercial (CC BY-NC 4.0) license, which permits others to distribute, remix, adapt, build upon this work non-commercially, and license their derivative works on different terms, provided the original work is properly cited, appropriate credit is given, any changes made indicated, and the use is non-commercial. See: http://creativecommons.org/licenses/by-nc/4.0/.

ORCID iDs

Mathew Baldwin http://orcid.org/0000-0002-8790-9975

Jonathan Alistair Cook http://orcid.org/0000-0002-4156-6989

\section{REFERENCES}

1 Linsell L, Dawson J, Zondervan K, et al. Prevalence and incidence of adults consulting for shoulder conditions in UK primary care; patterns of diagnosis and referral. Rheumatology 2006;45:215-21.

2 van der Windt DA, Koes BW, Boeke AJ, et al. Shoulder disorders in general practice: prognostic indicators of outcome. Br J Gen Pract 1996;46:519-23.

3 Virta L, Joranger P, Brox Jl, et al. Costs of shoulder pain and resource use in primary health care: a cost-of-illness study in Sweden. BMC Musculoskelet Disord 2012;13:17.

4 Murphy RJ, Carr AJ. Shoulder pain. BMJ Clin Evid 2010;2010:1107.

5 Sher JS, Uribe JW, Posada A, et al. Abnormal findings on magnetic resonance images of asymptomatic shoulders. J Bone Joint Surg Am 1995;77:10-15.

6 Yamamoto A, Takagishi K, Osawa T, et al. Prevalence and risk factors of a rotator cuff tear in the general population. J Shoulder Elbow Surg 2010;19:116-20.

7 Carr A, Cooper C, Campbell MK, et al. Effectiveness of open and arthroscopic rotator cuff repair (UKUFF). Bone Joint J 2017;99B:107-15.

8 Judge A, Murphy RJ, Maxwell R, et al. Temporal trends and geographical variation in the use of subacromial decompression and rotator cuff repair of the shoulder in England. Bone Joint $J$ 2014;96B:70-4.

9 Rashid MS, Cooper C, Cook J, et al. Increasing age and tear size reduce rotator cuff repair healing rate at 1 year. Acta Orthop 2017;88:606-11.

10 Ozaki J, Fujimoto S, Masuhara K, et al. Reconstruction of chronic massive rotator cuff tears with synthetic materials. Clin Orthop Relat Res 1986;202:173-83.

11 Shea KP, Obopilwe E, Sperling JW, et al. A biomechanical analysis of gap formation and failure mechanics of a xenograft-reinforced rotator cuff repair in a cadaveric model. J Shoulder Elbow Surg 2012;21:1072-9.

12 Shea KP, McCarthy MB, Ledgard F, et al. Human tendon cell response to 7 commercially available extracellular matrix materials: an in vitro study. Arthroscopy 2010;26:1181-8.

13 Mori D, Funakoshi N, Yamashita F. Arthroscopic surgery of irreparable large or massive rotator cuff tears with low-grade fatty degeneration of the infraspinatus: patch autograft procedure versus partial repair procedure. Arthroscopy 2013;29:1911-21.

14 Sedrakyan A, Campbell B, Merino JG, et al. IDEAL-D: a rational framework for evaluating and regulating the use of medical devices. BMJ 2016;353:i2372-7. 
15 Malcarney HL, Bonar F, Murrell GAC. Early inflammatory reaction after rotator cuff repair with a porcine small intestine submucosal implant. Am J Sports Med 2005;33:907-11.

16 Greenall G, Carr A, Beard D, et al. Systematic review of the surgical management of rotator cuff repair with an augmentative patch: a feasibility study protocol. Syst Rev 2018;7:187.

17 DeOrio JK, Cofield RH. Results of a second attempt at surgical repai of a failed initial Rotator-cuff repair. The Journal of Bone \& Joint Surgery 1984;66:563-7.

18 Gerber C, Fuchs B, Hodler J. The results of repair of massive tears of the rotator cuff. J Bone Joint Surg Am 2000;82:505-15.

19 Ferguson DP, Lewington MR, Smith TD, et al. Graft utilization in the augmentation of Large-to-Massive rotator cuff repairs. Am J Sports Med 2016;44:2984-92.

20 Coghlan JA, Buchbinder R, Green S, et al. Surgery for rotator cuff disease. Cochrane Database Syst Rev 2008:CD005619.

21 Thangarajah T, Pendegrass CJ, Shahbazi S, et al. Augmentation of rotator cuff repair with soft tissue scaffolds. Orthop J Sports Med 2015;3:232596711558749.

22 Bailey JR, Kim C, Alentorn-Geli E, et al. Rotator cuff matrix augmentation and interposition: a systematic review and metaanalysis. Am J Sports Med 2018;036354651877476.

23 Steinhaus ME, Makhni EC, Cole BJ, et al. Outcomes after patch use in rotator cuff repair. Arthroscopy 2016;32:1676-90.

24 Papalia R, Franceschi F, Zampogna B, et al. Augmentation techniques for rotator cuff repair. Br Med Bull 2013;105:107-38.

25 Higgins JPT, Altman DG, Gøtzsche PC, et al. The Cochrane Collaboration's tool for assessing risk of bias in randomised trials. BMJ 2011;343:d5928.

26 Sterne JAC, Hernán MA, Reeves BC, et al. ROBINS-I: a tool for assessing risk of bias in non-randomised studies of interventions. BMJ 2016;355:i4919.

27 Shamseer L, Moher D, Clarke M, et al. Preferred reporting items for systematic review and meta-analysis protocols (PRISMA-P) 2015 elaboration and explanation. BMJ 2015;349:g7647-25.

28 Cook JA, Merritt N, Rees JL, et al. Patch-augmented rotator cuff surgery (PARCS) study - protocol for a feasibility study. Pilot Feasibility Stud 2018;4:188

29 Leuzinger J, Sternberg C, Smolen D, et al. [Patch Augmentation in Rotator Cuff Repair Surgery with Elder Patients]. Z Orthop Unfall 2016;154:504-12.

30 Ciampi P, Scotti C, Nonis A, et al. The benefit of synthetic versus biological patch augmentation in the repair of posterosuperior massive rotator cuff tears. Am J Sports Med 2014:42:1169-75.

31 Maillot C, Harly E, Demezon H, et al. Surgical repair of large-tomassive rotator cuff tears seems to be a better option than patch augmentation or débridement and biceps tenotomy: a prospective comparative study. J Shoulder Elbow Surg 2018;27:1545-52.

32 Veen EJD, Diercks RL, Landman EBM, et al. The results of using a tendon autograft as a new rotator cable for patients with a massive rotator cuff tear: a technical note and comparative outcome analysis. J Orthop Surg Res 2020;15:1-7.

33 Lamas JR, García-Fernández C, Tornero-Esteban P, et al. Adverse effects of xenogenic scaffolding in the context of a randomized double-blind placebo-controlled study for repairing full-thickness rotator cuff tears. Trials 2019;20:1-9.

34 Walton JR, Bowman NK, Khatib Y, et al. Restore orthobiologic implant: not recommended for augmentation of rotator cuff repairs. $J$ Bone Joint Surg Am 2007;89:786-91.

35 Bryant $\mathrm{D}$, Holtby $\mathrm{R}$, Willits $\mathrm{K}$, et al. A randomized clinical trial to compare the effectiveness of rotator cuff repair with or without augmentation using porcine small intestine submucosa for patients with moderate to large rotator cuff tears: a pilot study. J Shoulder Elbow Surg 2016;25:1623-33.

36 Barber FA, Burns JP, Deutsch A, et al. A prospective, randomized evaluation of acellular human dermal matrix augmentation for arthroscopic rotator cuff repair. Arthroscopy 2012;28:8-15.

37 Avanzi P, Giudici LD, Capone A, et al. Prospective randomized controlled trial for patch augmentation in rotator cuff repair: 24-month outcomes. J Shoulder Elbow Surg 2019;28:1918-27.

38 Cai Y-Z, Zhang C, Jin R-L, et al. Arthroscopic rotator cuff repair with graft augmentation of 3-dimensional biological collagen for moderate to large tears: a randomized controlled study. Am J Sports Med 2018;46:1424-31.

39 lannotti JP, Codsi MJ, Kwon YW, et al. Porcine small intestine submucosa augmentation of surgical repair of chronic two-tendon rotator cuff tears. J Bone Joint Surg Am 2006;88:1238-44.

40 Gilot GJ, Alvarez-Pinzon AM, Barcksdale L, et al. Outcome of large to massive rotator cuff tears repaired with and without extracellular matrix augmentation: a prospective comparative study. Arthroscopy 2015;31:1459-65.
41 Flury M, Rickenbacher D, Jung C, et al. Porcine Dermis Patch Augmentation of Supraspinatus Tendon Repairs: A Pilot Study Assessing Tendon Integrity and Shoulder Function 2 Years After Arthroscopic Repair in Patients Aged 60 Years or Older. Arthroscopy 2018;34:24-37.

42 Ito J, Morioka T. Surgical treatment for large and massive tears of the rotator cuff. Int Orthop 2003;27:228-31.

43 Jeon YS, Lee J, Kim RG, et al. Does additional biceps augmentation improve rotator cuff healing and clinical outcomes in anterior Lshaped rotator cuff tears? clinical comparisons with arthroscopic partial repair. Am J Sports Med 2017;45:2982-8.

44 Tempelaere C, Desmoineaux P, Lespagnol F, et al. Surgical repair of massive rotator cuff tendon tears: autologous quadriceps tendon graft versus arthroscopic repair. Orthop Traumatol Surg Res 2017:103:435-40.

45 Yoon JP, Chung SW, Kim JY, et al. Outcomes of combined bone marrow stimulation and patch augmentation for massive rotator cuff tears. Am J Sports Med 2016;44:963-71.

46 Vitali M, Cusumano A, Pedretti A, et al. Employment of synthetic patch with augmentation of the long head of the biceps tendon in irreparable lesions of the rotator cuff. Tech Hand Up Extrem Surg 2015;19:32-9.

47 Phipatanakul WP, Petersen SA. Porcine small intestine submucosa xenograft augmentation in repair of massive rotator cuff tears. Am J Orthop 2009;38:572-5

48 Scheibel M, Brown A, Woertler K, et al. Preliminary results after rotator cuff reconstruction augmented with an autologous periosteal flap. Knee Surg Sports Traumatol Arthrosc 2007;15:305-14.

49 Schlegel TF, Abrams JS, Bushnell BD, et al. Radiologic and clinical evaluation of a bioabsorbable collagen implant to treat partialthickness tears: a prospective multicenter study. J Shoulder Elbow Surg 2018;27:242-51.

50 Moore DR, Cain EL, Schwartz ML, et al. Allograft reconstruction for massive, irreparable rotator cuff tears. Am J Sports Med 2006;34:392-6.

51 Tashjian RZ, Deloach J, Porucznik CA, et al. Minimal clinically important differences (MCID) and patient acceptable symptomatic state (pass) for visual analog scales (vas) measuring pain in patients treated for rotator cuff disease. J Shoulder Elbow Surg 2009;18:927-32.

52 Wylie JDet al. Functional outcomes assessment in shoulder surgery. World J Orthop 2014;5:623-33.

$53 \mathrm{Xu} \mathrm{S}$, Chen JY, Lie HME, et al. Determination of threshold scores for treatment success after arthroscopic rotator cuff repair using Oxford, constant, and University of California, Los Angeles shoulder scores. Arthroscopy 2019;35:304-11.

54 Berry MG, Stanek JJ. The PIP mammary prosthesis: a product recall study. J Plast Reconstr Aesthet Surg 2012;65:697-704.

55 Hirst A, Philippou Y, Blazeby J, et al. No surgical innovation without evaluation: evolution and further development of the ideal framework and recommendations. Ann Surg 2019;269:211-20.

56 Mori D, Funakoshi N, Yamashita F, et al. Effect of fatty degeneration of the infraspinatus on the efficacy of arthroscopic patch autograft procedure for large to massive rotator cuff tears. Am J Sports Med 2015;43:1108-17.

57 Agrawal V. Healing rates for challenging rotator cuff tears utilizing an acellular human dermal reinforcement graft. Int J Shoulder Surg 2012;6:36-44.

58 Audenaert E, Van Nuffel J, Schepens A, et al. Reconstruction of massive rotator cuff lesions with a synthetic interposition graft: a prospective study of 41 patients. Knee Surg Sports Traumatol Arthrosc 2006;14:360-4.

59 Badhe SP, Lawrence TM, Smith FD, et al. An assessment of porcine dermal xenograft as an augmentation graft in the treatment of extensive rotator cuff tears. J Shoulder Elbow Surg 2008;17:S35-9.

60 Bektaser Bet al. Free coracoacromial ligament graft for augmentation of massive rotator cuff tears treated with mini-open repair. Acta Orthop Traumatol Turc 2010;44:426-30.

61 Bond JL, Dopirak RM, Higgins J, et al. Arthroscopic replacement of massive, irreparable rotator cuff tears using a GraftJacket allograft: technique and preliminary results. Arthroscopy 2008;24:403.e1-403. e8.

62 Burkhead WZ, Schiffern SC, Krishnan SG. Use of graft jacket as an augmentation for massive rotator cuff tears. Semin Arthroplasty 2007:18:11-18.

63 Cho $\mathrm{C}-\mathrm{H}$, Lee S-M, Lee Y-K, et al. Mini-open suture bridge repair with porcine dermal patch augmentation for massive rotator cuff tear: surgical technique and preliminary results. Clin Orthop Surg 2014;6:329-35.

64 Consigliere P, Polyzois I, Sarkhel T, et al. Preliminary Results of a Consecutive Series of Large \& Massive Rotator Cuff Tears Treated 
with Arthroscopic Rotator Cuff Repairs Augmented with Extracellular Matrix. Arch Bone Jt Surg 2017;5:14-21.

65 Encalada-Diaz I, Cole BJ, Macgillivray JD, et al. Rotator cuff repair augmentation using a novel polycarbonate polyurethane patch: preliminary results at 12 months' follow-up. J Shoulder Elbow Surg 2011;20:788-94.

66 Flury M. [Arthroscopic rotator cuff repair with patch augmentation]. Oper Orthop Traumatol 2012;24:486-94.

67 Giannotti S, Ghilardi M, Dell'osso G, et al. Study of the porcine dermal collagen repair patch in morpho-functional recovery of the rotator cuff after minimum follow-up of 2.5 years. Surg Technol Int 2014;24:348-52.

68 Gouk CJC, Shulman RM, Buchan C, et al. Failure of dermal allograft repair of massive rotator cuff tears in magnetic resonance imaging and clinical assessment. Clin Orthop Surg 2019;11:200-7.

69 Gupta AK, Hug K, Berkoff DJ, et al. Dermal tissue allograft for the repair of massive irreparable rotator cuff tears. Am J Sports Med 2012;40:141-7.

70 Gupta AK, Hug K, Boggess B, et al. Massive or 2-tendon rotator cuff tears in active patients with minimal glenohumeral arthritis: clinical and radiographic outcomes of reconstruction using dermal tissue matrix xenograft. Am J Sports Med 2013;41:872-9.

71 Hirooka A, Yoneda M, Wakaitani S, et al. Augmentation with a GoreTex patch for repair of large rotator cuff tears that cannot be sutured. Journal of Orthopaedic Science 2002;7:451-6.

72 Johnson SM, Cherry J V, Thomas N, et al. Clinical outcomes and ultrasonographic viability of GraftJacket ${ }^{\circledR}$ augmented rotator cuff repair: a prospective follow-up study with mean follow-up of fortyone months. J Clin Orthop Trauma 2019:1-6.

73 Lederman ES, Toth AP, Nicholson GP, et al. A prospective, multicenter study to evaluate clinical and radiographic outcomes in primary rotator cuff repair reinforced with a xenograft dermal matrix. $J$ Shoulder Elbow Surg 2016;25:1961-70.

74 Lenart BA, Martens KA, Kearns KA, et al. Treatment of massive and recurrent rotator cuff tears augmented with a poly-l-lactide graft, a preliminary study. J Shoulder Elbow Surg 2015;24:915-21.

75 Marberry TA. A synthetic reinforcement patch in repair of challenging two-tendon rotator cuff tears. Shoulder Elbow 2013;5:24-9.

76 Metcalf MH, Savoie FH, Kellum B. Surgical technique for xenograft (SIS) augmentation of Rotator-cuff repairs. Oper Tech Orthop 2002;12:204-8.

77 Modi A, Singh HP, Pandey R, et al. Management of irreparable rotator cuff tears with the Graftjacket allograft as an interpositional graft. Shoulder \& Elbow 2013;5:188-94.
78 Nada AN, Debnath UK, Robinson DA, et al. Treatment of massive Rotator-cuff tears with a polyester ligament (Dacron) augmentation. $J$ Bone Joint Surg Br 2010;92-B:1397-402.

79 Neumann JA, Zgonis MH, Rickert KD, et al. Interposition dermal matrix xenografts: a successful alternative to traditional treatment of massive rotator cuff tears. Am J Sports Med 2017;45:1261-8.

80 Petrie MJ, Ismaiel AH. Treatment of massive Rotator-cuff tears with a polyester ligament (LARS) patch. Acta Orthop Belg 2013;79:620-5.

81 Petri M, Warth RJ, Horan MP, et al. Outcomes after open revision repair of massive rotator cuff tears with biologic patch augmentation. Arthroscopy 2016;32:1752-60.

82 Petriccioli D, Bertone C, Marchi G, et al. Open repair of isolated traumatic subscapularis tendon tears with a synthetic soft tissue reinforcement. Musculoskelet Surg 2013;97 Suppl 1:63-8.

83 Proctor CS. Long-Term successful arthroscopic repair of large and massive rotator cuff tears with a functional and degradable reinforcement device. J Shoulder Elbow Surg 2014;23:1508-13.

84 Rhee YG, Cho NS, Lim CT, et al. Bridging the gap in immobile massive rotator cuff tears: augmentation using the tenotomized biceps. Am J Sports Med 2008;36:1511-8.

85 Rotini R, Marinelli A, Guerra E, et al. Human dermal matrix scaffold augmentation for large and massive rotator cuff repairs: preliminary clinical and MRI results at 1-year follow-up. Musculoskelet Surg 2011;95:13-23.

86 Sano H, Mineta M, Kita A, et al. Tendon patch grafting using the long head of the biceps for irreparable massive rotator cuff tears. $J$ Orthop Sci 2010;15:310-6.

87 Sclamberg SG, Tibone JE, Itamura JM, et al. Six-Month magnetic resonance imaging follow-up of large and massive rotator cuff repairs reinforced with porcine small intestinal submucosa. J Shoulder Elbow Surg 2004;13:538-41.

88 Sears BW, Choo A, Yu A, et al. Clinical outcomes in patients undergoing revision rotator cuff repair with extracellular matrix augmentation. Orthopedics 2015;38:e292-6.

89 Smolen D, Haffner N, Mittermayr R, et al. Application of a new polyester patch in arthroscopic massive rotator cuff repair-a prospective cohort study. J Shoulder Elbow Surg 2020;29:e11-21.

90 Venouziou AI, Kokkalis ZT, Sotereanos DG. Human dermal allograft interposition for the reconstruction of massive irreparable rotator cuff tears. Am J Orthop 2013;42:63-70.

91 Wong I, Burns J, Snyder S. Arthroscopic GraftJacket repair of rotator cuff tears. J Shoulder Elbow Surg 2010;19:104-9.

92 Patte D. Classification of rotator cuff lesions. Clin Orthop Relat Res 1990;254:81-6. 